\title{
Variational Calculations of Nuclear Matter with Approximate Energy Expression
}

\author{
Kazunori Tanaka ${ }^{1}$ \\ RISE, Waseda University \\ 3-4-1, Okubo, Shinjuku-ku, Tokyo, 169-8555, Japan \\ E-mail: tanaka-kznr@aoni.waseda.jp \\ Masatoshi Takano \\ RISE, Waseda University \\ 3-4-1, Okubo, Shinjuku-ku, Tokyo, 169-8555, Japan \\ E-mail: takanom@waseda.jp
}

\begin{abstract}
The approximate energy expression for the variational calculation of nuclear matter is refined by taking into account three-body cluster terms with tensor correlations. In this variational method, the energy expression is constructed as an explicit functional of various two-body distribution functions which are regarded as variational functions. Then, the Euler-Lagrange equations are derived and fully minimized energies are obtained. The previous energy expression does not include the kinetic-energy terms caused by noncentral correlations sufficiently, and the obtained energy of nuclear matter is too low. Therefore, in this study, the energy expression is improved by taking into account important missing three-body-cluster kinetic-energy terms caused by tensor correlations. In this refinement, necessary conditions on tensor structure functions play important roles. The obtained energy per neutron for neutron matter with the v6' potential is considerably higher than that of the old energy expression.
\end{abstract}

10th Symposium on Nuclei in the Cosmos

Mackinac Island, Michigan, USA

27 July-1 August, 2008

\footnotetext{
$1 \quad$ Speaker
} 


\section{Introduction}

The equation of state (EOS) of nuclear matter is the crucial ingredient in neutron star studies, and the many-body approach starting from the realistic nuclear Hamiltonian provides us with a reliable nuclear EOS. We have been studying a variational method with approximate energy expressions.[1, 2] In this method, we construct the energy expression as an explicit functional of spin-isospin dependent radial, tensor and spin-orbit distribution functions which are regarded as variational functions. Then, the Euler-Lagrange equations are derived, and fully minimized energies are obtained. When we consider the two-body central force only, such as in liquid ${ }^{3} \mathrm{He}$, we obtain reasonable results with the variational method. However, in the case of nuclear matter in which the tensor and spin-orbit forces play important roles, the numerically calculated energies are too low, and the noncentral distribution functions have unrealistic long tails. The main reason for these unsatisfactory results is that the kinetic energy caused by the noncentral correlation is not included sufficiently in the energy expression. Therefore, in this study, we refine the energy expression with respect to the tensor correlation, as the first step of the refinement.

\section{Energy Expression for Neutron Matter}

In this paper, we consider neutron matter at zero temperature, and start from the following nuclear Hamiltonian;

$$
H=-\sum_{i=1}^{N} \frac{\hbar^{2}}{2 m} \nabla_{i}^{2}+\sum_{i<j}^{N} V_{i j} .
$$

Here, $m$ is the neutron mass, and the two-body potential $V_{i j}$ is written as

$$
V_{i j}=\sum_{s=0}^{1}\left\{V_{\mathrm{CS}}\left(r_{i j}\right)+s\left[V_{\mathrm{T}}\left(r_{i j}\right) S_{\mathrm{T} i j}+V_{\mathrm{SO}}\left(r_{i j}\right)\left(\boldsymbol{s} \cdot \boldsymbol{L}_{i j}\right)\right]+V_{\mathrm{qL} s}\left(r_{i j}\right) \boldsymbol{L}_{i j}^{2}+s V_{\mathrm{qSO}}\left(r_{i j}\right)\left(\boldsymbol{s} \cdot \boldsymbol{L}_{i j}\right)^{2}\right\} P_{s i j},
$$

where $S_{\mathrm{T} i j}$ is the tensor operator, $\boldsymbol{L}_{i j}$ is the orbital angular momentum operator and $P_{s i j}$ is the spin projection operator.

The energy expression is constructed with the radial distribution functions $F_{\mathrm{Cs}}(r)$, the tensor distribution function $F_{\mathrm{T}}(r)$ and the spin-orbit distribution function $F_{\mathrm{SO}}(r)$ as well as the structure functions $S_{\mathrm{C} 1}(k)$ and $S_{\mathrm{C} 2}(k)$, which are defined as

$$
\begin{aligned}
& F_{s}\left(r_{12}\right)=\Omega^{2} \sum_{\text {spin }} \int \Psi^{\dagger}\left(x_{1}, \cdots, x_{N}\right) P_{s 12} \Psi\left(x_{1}, \cdots, x_{N}\right) d \boldsymbol{r}_{3} \cdots d \boldsymbol{r}_{N}, \quad(s=0,1) \\
& F_{\mathrm{T}}\left(r_{12}\right)=\Omega^{2} \sum_{\text {spin }} \int \Psi^{\dagger}\left(x_{1}, \cdots, x_{N}\right) S_{\mathrm{T} 12} \Psi\left(x_{1}, \cdots, x_{N}\right) d \boldsymbol{r}_{3} \cdots d \boldsymbol{r}_{N},
\end{aligned}
$$




$$
\begin{gathered}
F_{\mathrm{SO}}\left(r_{12}\right)=\Omega^{2} \sum_{\text {spin }} \int \Psi^{\dagger}\left(x_{1}, \cdots, x_{N}\right)\left(\boldsymbol{s} \cdot \boldsymbol{L}_{12}\right) \Psi\left(x_{1}, \cdots, x_{N}\right) d \boldsymbol{r}_{3} \cdots d \boldsymbol{r}_{N}, \\
S_{\mathrm{C} 1}(k)=\frac{1}{N}\left\langle\left|\sum_{i=1}^{N} \exp \left(i \boldsymbol{k} \cdot \boldsymbol{r}_{i}\right)\right|^{2}\right\rangle=1+S_{1}(k)+S_{0}(k), \\
S_{\mathrm{C} 2}(k)=\frac{1}{3 N}\left\langle\left|\sum_{i=1}^{N} \boldsymbol{\sigma}_{i} \exp \left(i \boldsymbol{k} \cdot \boldsymbol{r}_{i}\right)\right|^{2}\right\rangle=1+\frac{1}{3} S_{1}(k)-S_{0}(k),
\end{gathered}
$$

where $\Omega$ is the volume of the system. In Eqs. (6) and (7), $S_{s}(k)(s=0,1)$ is

$$
S_{s}(k)=\rho \int\left[F_{s}(r)-F_{s}(\infty)\right] \exp (i \boldsymbol{k} \cdot \boldsymbol{r}) d \boldsymbol{r},
$$

with $\rho$ being the number density.

Using these functions and the auxiliary functions $F_{\mathrm{Cs}}(r), g_{\mathrm{T}}(r)$ and $g_{\mathrm{SO}}(r)$ defined in Ref. [2], the energy per neutron in neutron matter was constructed in Ref. [2] as follows:

$$
\begin{aligned}
& \frac{E_{\mathrm{old}}}{N}=\frac{3}{5} E_{\mathrm{F}}+2 \pi \rho \int_{0}^{\infty}\left\{\left[\sum_{s=0}^{1} F_{s}(r) V_{\mathrm{C} s}(r)\right]+F_{\mathrm{T}}(r) V_{\mathrm{T}}(r)+F_{\mathrm{SO}}(r) V_{\mathrm{SO}}(r)\right\} r^{2} d r \\
& +2 \pi \rho \int_{0}^{\infty}\left\{\left[\sum_{s=0}^{1} F_{\mathrm{qL} s}(r) V_{\mathrm{qL} s}(r)\right]+F_{\mathrm{q} S \mathrm{O}}(r) V_{\mathrm{qSO}}(r)\right\} r^{2} d r \\
& +\frac{\pi \hbar^{2} \rho}{2 m} \int_{0}^{\infty} \sum_{s=0}^{1}\left\{F_{\mathrm{C} s}(r)\left[\frac{1}{F_{\mathrm{C} s}(r)} \frac{d F_{\mathrm{C} s}(r)}{d r}-\frac{1}{F_{\mathrm{Fs}}(r)} \frac{d F_{\mathrm{Fs}}(r)}{d r}\right]^{2}\right\} r^{2} d r \\
& +\frac{2 \pi \hbar^{2} \rho}{m} \int_{0}^{\infty}\left\{8\left[\left\{\frac{d g_{\mathrm{T}}(r)}{d r}\right\}^{2}+6 \frac{\left\{g_{\mathrm{T}}(r)\right\}^{2}}{r^{2}}\right] F_{\mathrm{F} 1}(r)+\frac{2}{3}\left[\frac{d g_{\mathrm{SO}}(r)}{d r}\right]^{2} F_{\mathrm{qF} 1}(r)\right\} r^{2} d r+\frac{E_{\mathrm{CT}}}{N},(9)
\end{aligned}
$$

where

$$
\frac{E_{\mathrm{CT}}}{N}=-\frac{\hbar^{2}}{16 m \pi^{2} \rho} \int_{0}^{\infty} \sum_{n=1}^{2}(2 n-1) \frac{\left[S_{\mathrm{C} n}(k)-1\right]\left[S_{\mathrm{C} n}(k)-S_{\mathrm{CF}}(k)\right]^{2}}{S_{\mathrm{C} n}(k) / S_{\mathrm{CF}}(k)} k^{4} d k
$$

The first term on the right-hand side of Eq. (9) is the one-body kinetic-energy term with $E_{\mathrm{F}}$ being the Fermi energy. The second and the third terms are the potential energies, and the remaining terms are the kinetic energies caused by the correlation between neutrons. 


\section{Refinement of the Energy Expression for Neutron Matter}

In this section, we refine the kinetic-energy expression in Eq. (9) with respect to the tensor correlation using the cluster expansion method. First, we assume the Jastrow-type wave function

$$
\Psi\left(x_{1}, \cdots, x_{N}\right)=\operatorname{Sym}\left[\prod_{i<j} f_{i j}\right] \Phi\left(x_{1}, \cdots, x_{N}\right) .
$$

Here, Sym[ ] is the symmetrizer with respect to the order of the factors, $\Phi$ is the Fermi-gas wave function and $f_{i j}$ is the correlation function;

$$
f_{i j}=\sum_{s=0}^{1}\left[f_{\mathrm{Cs}}\left(r_{i j}\right)+s f_{\mathrm{T}}\left(r_{i j}\right) S_{\mathrm{T} i j}\right] P_{s i j}
$$

where $f_{\mathrm{Cs}}(r)$ and $f_{\mathrm{T}}(r)$ are the spin-dependent central and tensor correlation functions, respectively.

Then, we cluster-expand $\Delta E / N=\left\langle H>/ N-E_{\text {old }} / N\right.$ with $\langle H>$ being the expectation value of the Hamiltonian. Since the two-body cluster terms and the main part of the central-type threebody cluster terms in $\left\langle H>/ N\right.$ are completely included in $E_{\text {old }} / N$, the main part of $\Delta E / N$ is the three-body cluster terms including the tensor correlation $f_{\mathrm{T}}(r)$. We can express the main part of $\Delta E / N$ using the structure functions as

$$
\frac{\Delta E_{3}}{N}=-\frac{\hbar^{2}}{32 m \pi^{2} \rho} \int_{0}^{\infty}\left\{\left[S_{\mathrm{C} 2}(k)-1\right]\left[S_{\mathrm{T}}(k)\right]^{2}-\frac{1}{18}\left[S_{\mathrm{T}}(k)\right]^{3}\right\} k^{4} d k,
$$

where $S_{\mathrm{T}}(k)$ is the tensor structure function defined as

$$
S_{\mathrm{T}}(k)=4 \pi \rho \int_{0}^{\infty} F_{\mathrm{T}}(r) j_{2}(k r) r^{2} d r
$$

Here, we note that $\Delta E_{3} / N$ goes to negative infinity through the variational procedure. In order to convert this harmful term to a harmless one, we take into account the following necessary conditions on the tensor structure function:

$$
\begin{aligned}
& S_{\mathrm{CT} 1}(k)=\frac{1}{k^{2} N}\left\langle\left|\sum_{i=1}^{N}\left(\boldsymbol{\sigma}_{i} \cdot \boldsymbol{k}\right) \exp \left(i \boldsymbol{k} \cdot \boldsymbol{r}_{i}\right)\right|^{2}\right\rangle=S_{\mathrm{C} 2}(k)-\frac{1}{3} S_{\mathrm{T}}(k) \geq 0, \\
& S_{\mathrm{CT} 2}(k)=\frac{1}{2 k^{2} N}\left\langle\left|\sum_{i=1}^{N}\left(\boldsymbol{\sigma}_{i} \times \boldsymbol{k}\right) \exp \left(i \boldsymbol{k} \cdot \boldsymbol{r}_{i}\right)\right|^{2}\right\rangle=S_{\mathrm{C} 2}(k)+\frac{1}{6} S_{\mathrm{T}}(k) \geq 0 .
\end{aligned}
$$


Then, we refine the expression $E_{\mathrm{CT}} / N$ in Eq. (10) as follows:

$$
\begin{aligned}
\frac{E_{\mathrm{CTnew}}}{N}=-\frac{\hbar^{2}}{16 \pi^{2} m \rho} \int_{0}^{\infty}\left\{\frac{\left[S_{\mathrm{C} 1}(k)-1\right]\left[S_{\mathrm{C} 1}(k)-S_{\mathrm{CF}}(k)\right]^{2}}{S_{\mathrm{C} 1}(k) / S_{\mathrm{CF}}(k)}\right. \\
\left.+\sum_{n=1}^{2} n \frac{\left[S_{\mathrm{CT} n}(k)-1\right]\left[S_{\mathrm{CT} n}(k)-S_{\mathrm{CF}}(k)\right]^{2}}{S_{\mathrm{CT} n}(k) / S_{\mathrm{CF}}(k)}\right\} k^{4} d k .
\end{aligned}
$$

This term includes $\Delta E_{3} / N$ properly and guarantees the necessary conditions on the structure functions, Eqs. (6), (7), (15) and (16).

\section{Numerical calculations and discussion}

Using the refined energy expression, we calculate the energy per neutron in neutron matter with the v6' potential. As shown in Fig.1, the obtained energies are higher than those of the old energy expression. Although not shown here, the unrealistic long tail of $F_{\mathrm{T}}(r)$, which accompanied the old expression, disappears with the new energy expression. We note that $S_{\mathrm{CT} 2}(k)$ obtained with the old energy expression violates the inequality (16), which implies that the necessary conditions on the tensor structure function play important roles.

We also refined the energy expression for symmetric nuclear matter in a similar way. Numerical calculations of the energy for symmetric nuclear matter are now in progress.

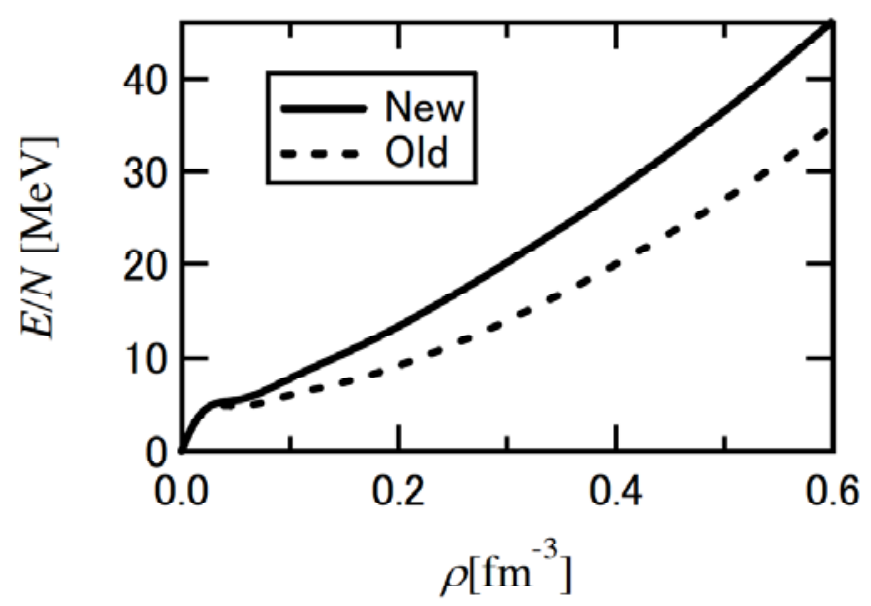

Figure 1: Energy per neutron for neutron matter with the v6' potential. The solid line is for the refined energy expression and the dashed line is for the old one.

\section{References}

[1] M. Takano and M. Yamada, Prog. Theor. Phys. 91, 1149(1994).

[2] M. Takano and M. Yamada, Prog. Theor. Phys. 100, 745(1998). 